\title{
Análisis biomecánico de la ligamentoplastía interespinosa con fibra de poliéster posterior a discectomía
}

\author{
Biomechanical analysis of interespinous ligamentoplasty with polyester fiber after discectomy \\ Martínez-López L,* Hermida-Ochoa JC, ${ }^{\ddagger}$ Hermida-Ochoa EH, ${ }^{\S}$ \\ Cuevas-Andrade JL, " Benavides-Rodríguez D"
}

Hospital de Ortopedia para Niños «Dr. Germán Díaz Lombardo».

RESUMEN. Introducción: Existen diferentes patologías de la columna lumbar que condicionan una inestabilidad biomecánica y clínica para su tratamiento, se han efectuado diversas técnicas de estabilización que tratan de preservar el movimiento y la transmisión de carga del segmento afecto como la ligamentoplastía interespinosa con fibra Dallos ${ }^{\circledR}$. Objetivo: Mostrar las variaciones biomecánicas de segmento funcional de columna lumbar de porcino, antes y después de realizar la discetomía y ligamentoplastía con fibra Dallos ${ }^{\circledR}$. Material y métodos: El segmento lumbar se montó en un simulador multiaxial servo-hidráulico. Se simularon rangos de movilidad de flexión, extensión, flexiones laterales y rotaciones axiales en tres condiciones: 1) segmento natural; 2) segmento discectomizado; y 3) segmento disectomizado más ligamentoplastía con fibra Dallos ${ }^{\circledR}$. Los rangos de movilidad se realizan hasta un torque de $7.5 \mathrm{~N}-\mathrm{m}$. La data de los torques y rangos de movilidad se recolectó en el programa del simulador y se graficaron los resultados de los cambios biomecánicos entre las tres condiciones descritas. Resultados: Se demostró que la
ABSTRACT. Introduction: There are different pathologies of the lumbar spine that condition a biomechanical and clinical instability for its treatment, various stabilization techniques have been carried out that try to preserve the movement and the transmission of load of the affected segment such as the interspinatus ligamentplasty with Dallos ${ }^{\circledR}$ fiber. Objective: To show the biomechanical variations of functional segment of lumbar spine of pigs, before and after performing the discectomy and ligamentoplasty with Dallos ${ }^{\circledR}$ fiber. Material and methods: The lumbar segment was mounted in a servohydraulic multiaxial simulator. Mobility ranges of flexion, extension, lateral flexion and axial rotations were simulated under three conditions: 1. Natural segment, 2. Discectomy segment, and 3. Disectomized segment plus ligamentoplasty with Dallos ${ }^{\circledR}$ fiber. The mobility ranges are made up to a torque of $7.5 \mathrm{~N}-\mathrm{m}$ The data of the torques and mobility ranges was collected in the simulator program and the results of the biomechanical changes between the three conditions described were plotted. Results: It was shown that lumbar

\section{Nivel de evidencia: IV}

* Residente de la Alta Especialidad en Cirugía de Columna Facultad Mexicana de Medicina, Universidad La Salle México con sede en el Hospital de Ortopedia para Niños «Dr. Germán Díaz Lombardo».

‡ Director del Centro de Investigación y Laboratorio de Biomecánica (CILAB) del Hospital de Ortopedia para Niños «Dr. Germán Díaz Lombardo».

$\S$ Director General y Cirujano de Columna del Hospital de Ortopedia para Niños «Dr. Germán Díaz Lombardo».

^ Maestro en Ciencias en Ingeniería Mecánica de la Sección de Estudios de Postgrado e Investigación ESIME Zacatenco, Instituto Politécnico Nacional.

" Subdirector y Cirujano de Columna del Hospital de Ortopedia para Niños «Dr. Germán Díaz Lombardo».

Correspondencia:

Lucio Martínez-López

Calle Carmen Núm. 18,

Col. Chimalistac, C.P. 01070

Alcaldía Álvaro Obregón, Ciudad de México.

E-mail: tyo_martinez@hotmail.com

Recibido: 22-01-2020. Aceptado: 15-01-2021.

Citar como: Martínez-López L, Hermida-Ochoa JC, Hermida-Ochoa EH, Cuevas-Andrade JL, Benavides-Rodríguez D. Análisis biomecánico de la ligamentoplastía interespinosa con fibra de poliéster posterior a discectomía. Acta Ortop Mex. 2021; 35(2): 125-131. https://dx.doi.org/10.35366/101854

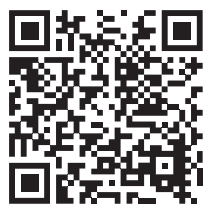


biomecánica lumbar es afectada después de la discectomía principalmente en la flexión y la extensión. En los movimientos de flexión y rotación axial izquierdas, se comprobó una alteración del torque y los rangos de movilidad. Conclusiones: La ligamentoplastía recupera parte de la estabilidad perdida postdiscectomía, preservando parte de la altura discal sin llegar a igualar los movimientos como en el segmento natural. Posterior a la discectomía, la distribución de la fuerza sugiere que la inestabilidad residual con ligamento plastía puede representar sobrecarga facetaria.

Palabras clave: Columna lumbar, inestabilidad, Dallos, ligamentoplastía, discectomía.

\section{Introducción}

Existen diferentes patologías de la columna lumbar que condicionan una inestabilidad biomecánica y clínica. ${ }^{1}$ Por afección del subsistema pasivo descrito por Panjabi y colaboradores principalmente de las estructuras disco-ligamentarias, para su tratamiento se han efectuado diversas técnicas de descompresión con y sin fusión, o bien por la estabilización dinámica, tratando de preservar el movimiento y la transmisión de carga del segmento afecto. ${ }^{2,}$

Posner y White definieron a la inestabilidad clínica como «...la pérdida de la capacidad de la columna, bajo cargas fisiológicas, para mantener un patrón normal de desplazamiento, de tal manera que no se produzca déficit neurológico, deformidades mayores ni dolor incapacitante». ${ }^{4}$ Asimismo, la inestabilidad biomecánica fue definida por Kirkaldy y colaboradores. ${ }^{5}$

Un ejemplo de inestabilidad es la espondilolistesis lumbar, en donde el estándar para su tratamiento ha sido la fijación rígida con artrodesis. ${ }^{6}$ Sin embargo se ha observado que puede llegar a ocasionar degeneración del segmento adyacente y presencia de enfermedad del segmento adyacente. ${ }^{7}$ Alpízar y su grupo evaluaron la aparición de degeneración del segmento adyacente y la presencia de enfermedad del segmento adyacente en la estabilización dinámica interespinosa versus fijación transpedicular y artrodesis en el tratamiento del conducto lumbar estrecho, encontrando una incidencia de aparición de degeneración del segmento adyacente de $33.3 \%$ en el grupo de la fijación con artrodesis y $20 \%$ en el grupo de la ligamentoplastía interespinosa (ILP) a los tres años de seguimiento. ${ }^{8}$

Okuda y su equipo encontraron en su estudio sobre la enfermedad del segmento adyacente después de la fusión intersomática lumbar posterior de un solo segmento para la espondilolistesis degenerativa a 10 años de seguimiento una incidencia radiográfica de $75 \%$, sintomático de $31 \%$ y quirúrgico de $15 \%$ en PLIF. ${ }^{9}$

Debido a que se ha observado una alta incidencia de la enfermedad del segmento adyacente en la función lumbar, los cirujanos de columna han optado por la estabilización biomechanics is affected after discectomy mainly in flexion and extension. In the left axial bending and rotation movements, an alteration of torque and mobility ranges was found. Conclusions: The ligamentoplasty recovers part of the stability lost after discectomy preserving part of the disc height without reaching to equalize the movements as in the natural segment. After discectomy the distribution of force suggests that residual instability with ligament plasty may represent facet overload.

Keywords: Lumbar spine, instability, Dallos, ligamentoplasty, discectomy.

dinámica como alternativa de tratamiento y restablecer la zona neutra de la columna. ${ }^{10,11}$

Dentro de los dispositivos de estabilización dinámica en el área de la enfermedad de la columna lumbar, tenemos como ejemplos de implantes interespinosos el dispositivo DIAM (Metronic), Wallis (Spain Next), Minns, In-Space (Synthes). ${ }^{12,13,14,15}$

La ligamentoplastía interespinosa (ILP) es un ejemplo de estabilización dinámica y fue introducido por primera vez por Senegas y colaboradores para el tratamiento de la espondilolistesis degenerativa después de una laminectomía extensa, restringiendo la flexión de la columna lumbar preservando el ligamento interespinoso y supraespinoso. ${ }^{16}$

En la actualidad, el uso del ligamento de poliéster (Dallos ${ }^{\circledR}$ ) en la ligamentoplastía interespinosa se extiende a diversas patologías de la columna lumbar después de una fijación y fusión vertebral, tras una discectomía clásica, espondilolistesis degenerativa, inestabilidad angulatoria de

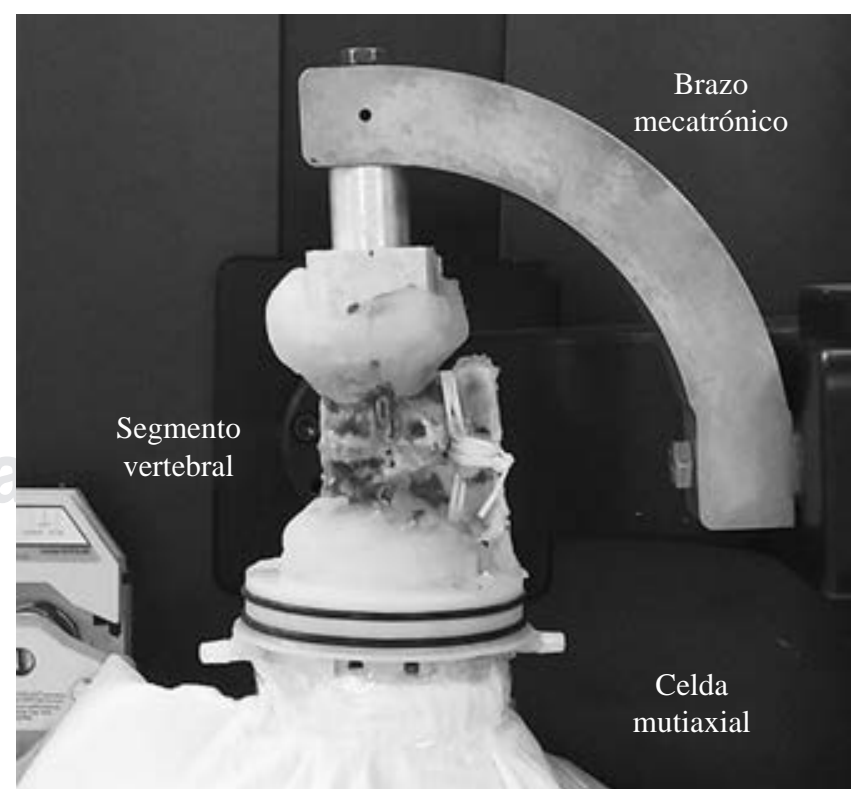

Figura 1: Montaje del segmento lumbar en posición anatómica neutra en el simulador servo-hidráulico. 


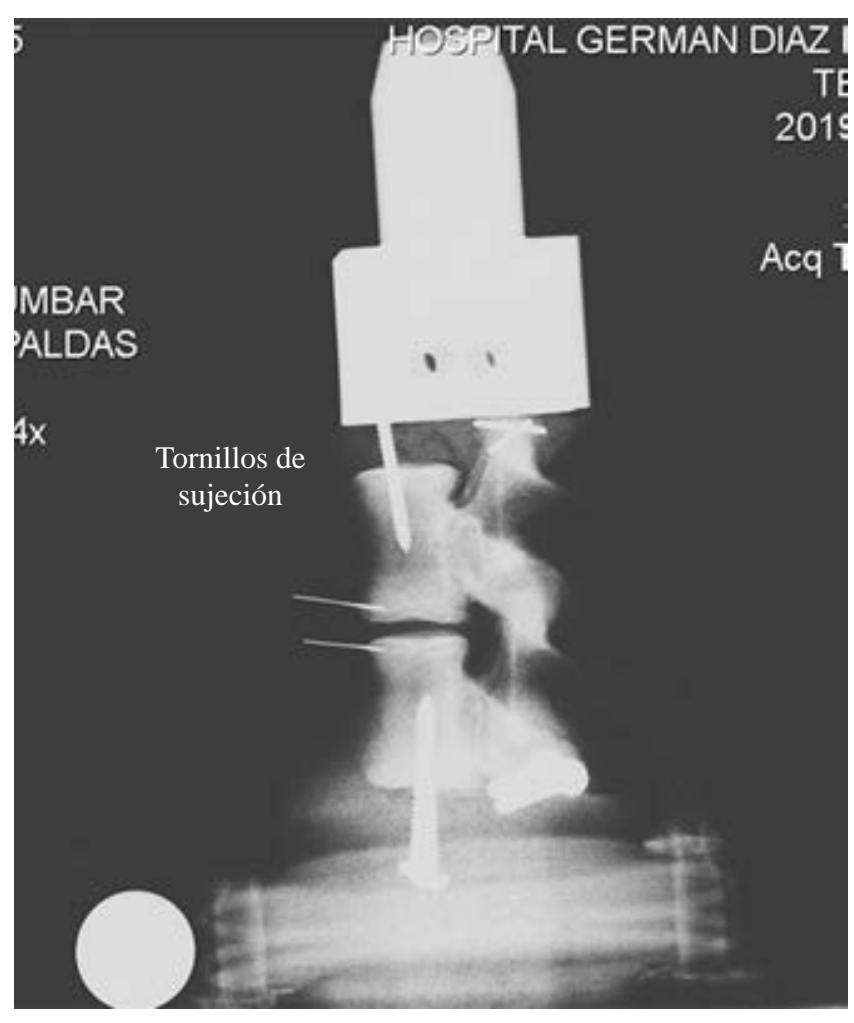

Figura 2: Radiografía que muestra la fijación del segmento lumbar en posición neutra, cementado superior e inferior mediante una capa de PMMA.

L1 a S1, reconstrucción de los ligamentos interespinosos y supraespinosos, conducto lumbar estrecho y prevención del segmento adyacente en la artrodesis o fijación rígida con la finalidad de restablecer la zona neutra. ${ }^{7,8,17}$

Hong y su grupo demostraron que el uso de la ligamentoplastía interespinosa como tratamiento de la espondilolistesis, en donde los pacientes presentaron mejoría clínica significativa y en las radiografías dinámicas, el movimiento angular fue similar entre las mediciones de seguimiento preoperatorias y más recientes, pero el movimiento traslacional disminuyó significativamente. ${ }^{18}$ Rosales y colaboradores en un análisis clínico y radiográfico encontraron que la ligamentoplastía interespinosa con ligamento Dallos ${ }^{\circledR}$ mejora la estabilidad segmentaria, permite la movilidad dentro de los rangos normales, conserva la altura discal a los cuatro años de seguimiento y ofrece una mayor estabilidad dinámica con una notable mejoría clínica. ${ }^{19}$

Existen varios estudios con resultados favorables sobre el uso de la ligamentoplastía interespinosa desde el punto de vista clínico y radiográfico, pero no biomecánico. El presente estudio se centra en el análisis biomecánico de la ligamentoplastía interespinosa con una fibra sintética de poliéster realizado en un segmento lumbar porcino fresco en tres diferentes condiciones: segmento natural, inestable (discectomía) y con la ligamentoplastía interespinosa, analizando los rangos de movimiento en flexión, extensión, flexión derecha e izquierda, así como la rotación derecha e izquierda primero con una carga de $400 \mathrm{~N}$.

\section{Material y métodos}

Obtención y preparación del segmento lumbar: se utilizó un segmento de columna lumbar de porcino obtenido en un establecimiento que cuenta con la certificación de la NOM-033-ZOO-1995, después el segmento fue conservado a $-26^{\circ}$ y descongelado a temperatura ambiente inmediatamente antes de realizar su montaje en el simulador.

Se realizó una disección minuciosa del segmento lumbar retirando la masa muscular paravertebral, preservando la integridad del ligamento interespinoso y el supraespinoso, cápsula articular de las facetas, ligamentos longitudinales y disco intervertebral. La fijación del segmento se realizó en sus dos polos, en el polo inferior se colocaron dos tornillos de forma divergente que atravesó la plataforma inferior la mitad del cuerpo de la vértebra infrayacente, posteriormente se hizo el montaje en una plataforma prediseñada para el simulador servo-hidráulico, mecatrónico articular (VIVO, Force and Motion de AMTI, Waltham MA) con PMMA integrando su vez a la faceta articular interior (Figura 1).

El polo superior se fijó con dos tornillos de forma divergente que atravesaron la plataforma superior y mitad del cuerpo, finalmente se fijó a un adaptador de aluminio con PMMA integrando también a la faceta articular superior (Figura 2).

Montaje del segmento: el montaje se realizó en nuestro laboratorio de biomecánica en un simulador servo-hidraúlico, mecatrónico articular con seis ejes de libertad representando fuerza y movimientos rotacionales en los ejes X, Y y Z (VIVO, Force and Motion de AMTI, Waltham MA). La plataforma inferior cuenta con una celda que registra cargas y momentos en todos los ejes, así como translación superior e inferior en el eje Z, translación anteroposterior del eje Y y medio-lateral del eje $X$. Cuenta con un brazo mecatrónico de flexoextensión para el eje X y otro para el eje Y para simular las flexiones laterales derecha e izquierda. La placa inferior tiene la capacidad de rotar en ambos sentidos para recrear la rotación lateral derecha e izquierda.

Una vez fijados ambos polos del segmento a los adaptadores prediseñados para el simulador servo-hidráulico multiaxial, se buscó su equilibrio vertical e iniciaron las pruebas en los diferentes ROM, la medición se realizó mediante las celdas integradas en la plataforma inferior, los datos fueron colectados en la computadora que contiene el software del fabricante de la máquina.

Técnica de la ligamentoplastía con fibra Dallos ${ }^{\circledR}$ se siguió el método descrito por el fabricante, el cual se realizó por un cirujano de columna experimentado de nuestro servicio (Figura 3).

Prueba biomecánica: una vez montado el espécimen en el simulador servo-hidráulico, se realizaron pruebas biomecánicas en tres diferentes condiciones: 1) segmento natural intacto que consistió de uno lumbar sin musculatura vertebral, pero preservando los complejos ligamentarios, incluyendo los ligamentos inter- y supraespinosos y las cápsulas facetarias; 2) segmento posterior a discectomía mediante 

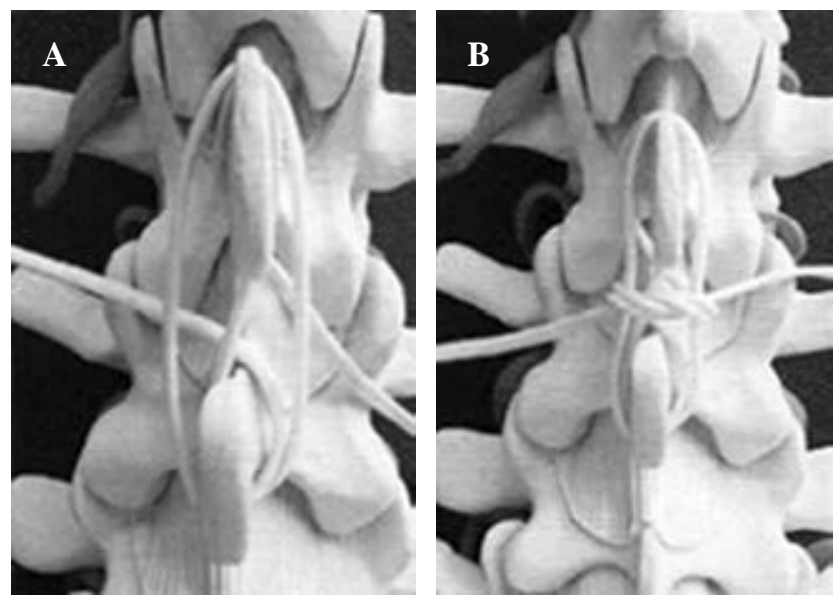

Flexión 400N (desplazamiento mm)

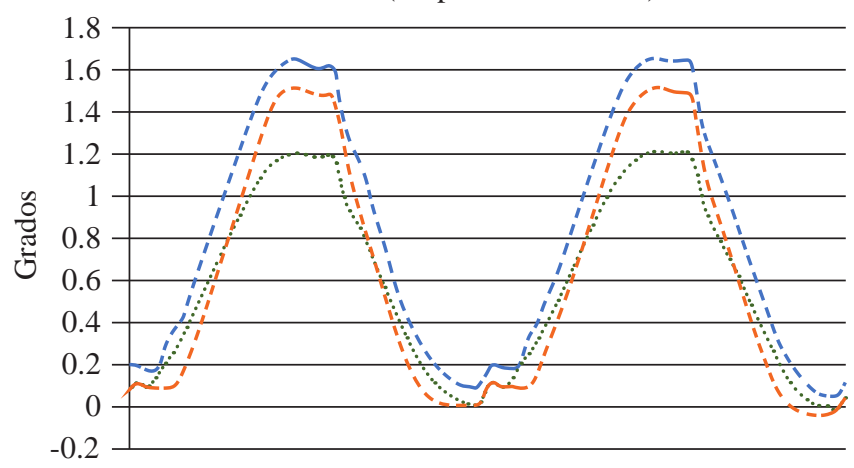

A Torque (N-m)

Flexión natural - - _ - Flexión nodisc Flexión dallos

Extensión 400N (desplazamento mm)

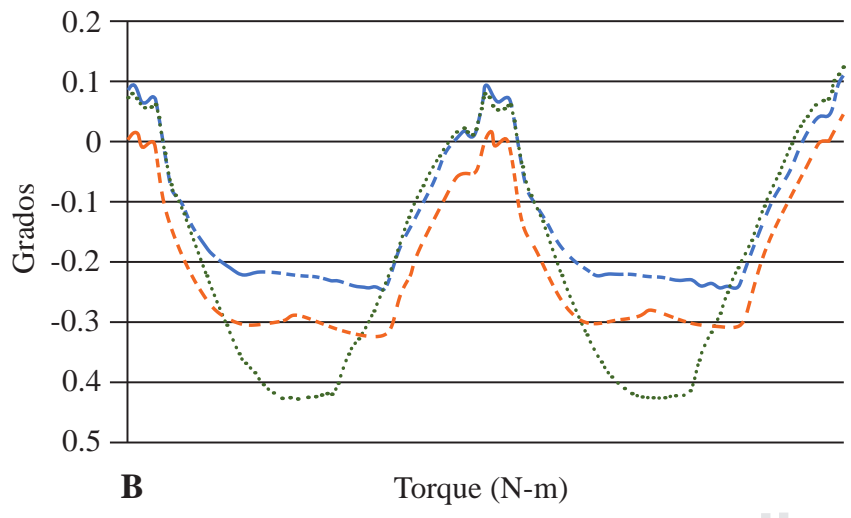

Extensión natural _ _ _ _ Extensión nodisc _____ Extensión dallos

Figura 4: A) Muestra la flexión, registrando el aumento de los arcos de movilidad para obtener el torque de $7.5 \mathrm{~N}$-m en la condición sin disco, misma que muestra una recuperación significativa después de la ligamentoplastía. B) Análisis de la extensión, muestra el mismo fenómeno que la flexión más el aplanamiento de las curvas en las condiciones sin disco y sin disco más ligamentoplastía, ilustrando la pérdida del comportamiento viscoelástico después de la resección del disco más el choque con los elementos óseos posteriores compuestos por las articulaciones cigapofisarias y las apófisis espinosas. La curva de extensión con disco intacto muestra una curva sinusoidal continua que representa la viscoelasticidad uniforme que confiere el disco.

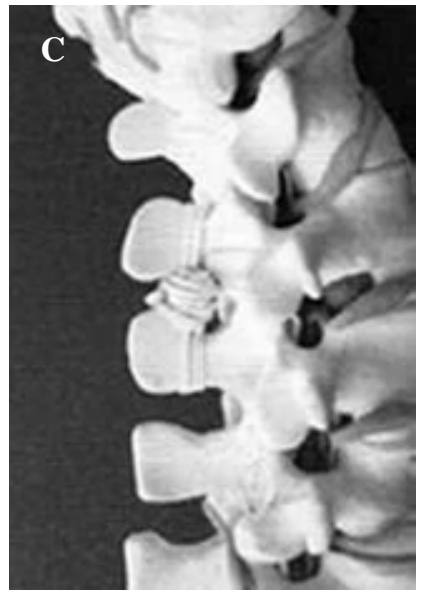

Figura 3:

Técnica de colocación de la ligamentoplastía interespinosa con fibra Dallos ${ }^{\circledR}$. acceso posterior izquierdo con laminectomía parcial; y 3) segmento con discectomía más ligamentoplastía mediante ligamento de poliéster $\left(\right.$ Dallos $\left.^{\circledR}\right)$. Cada condición se sujetó a seis pruebas diferentes consistentes en flexión, extensión, flexión lateral derecha e izquierda y rotación derecha e izquierda con precarga de acondicionamiento de $20 \mathrm{~N}$. Después estas seis condiciones se repitieron bajo una carga de $400 \mathrm{~N}$ para obtener una cantidad total de 36 pruebas. El movimiento en todas direcciones fue prescrito mediante desplazamiento gradual hasta obtener un torque de $7.5 \mathrm{~N}-\mathrm{m}$ como lo establece el protocolo estándar de pruebas en segmentos de columna para dispositivos de columna (Panjabi). Una vez establecido el torque y el rango de movilidad en las diferentes direcciones, se realizaron 10 ciclos de cada evento a una frecuencia de $1 \mathrm{~Hz}$. Las curvas de desplazamiento vs torque en todas las direcciones fueron registradas mediante el software del simulador, graficadas en Excel y comparadas entre las tres condiciones mencionadas para establecer el grado de estabilidad e inestabilidad entre éstas. También se registró el desplazamiento axial en mm de la placa inferior para definir los cambios de altura del espacio intervertebral.

\section{Resultados}

Los grados para alcanzar el torque recomendado de 7.5 $\mathrm{N}-\mathrm{m}$ sin carga axial en flexión para las condiciones de segmento natural, postdiscectomía y postdiscectomía + ligamentoplastía fueron de $7.28^{\circ}, 8.77^{\circ}$ y $7.37^{\circ}$ respectivamente (Figura 4A).

Para la extensión, los grados fueron de $1.19^{\circ}, 2.11^{\circ} \mathrm{y}$ $1.23^{\circ}$ (Figura 4B).

Para la flexión lateral derecha, los grados fueron de $12.1^{\circ}$, $12.5^{\circ}$ y $11.9^{\circ}$ (Figura $5 B$ ) y para la izquierda $15.4^{\circ}, 26.7^{\circ}$ y $35.4^{\circ}$ (Figura 5A).

Por último, los grados para la rotación derecha fueron $1.4^{\circ}, 1.4^{\circ}$ y $1.2^{\circ}$ (Figura 6 A) y para la izquierda $2.67^{\circ}, 2.68^{\circ}$ y $2.7^{\circ}$ (Figura $6 B$ ).

Los grados para alcanzar el torque recomendado de $7.5 \mathrm{~N}-\mathrm{m}$ para las pruebas bajo carga de $400 \mathrm{~N}$ en las tres con- 
Flexión lateral izquierda 400N (grados)

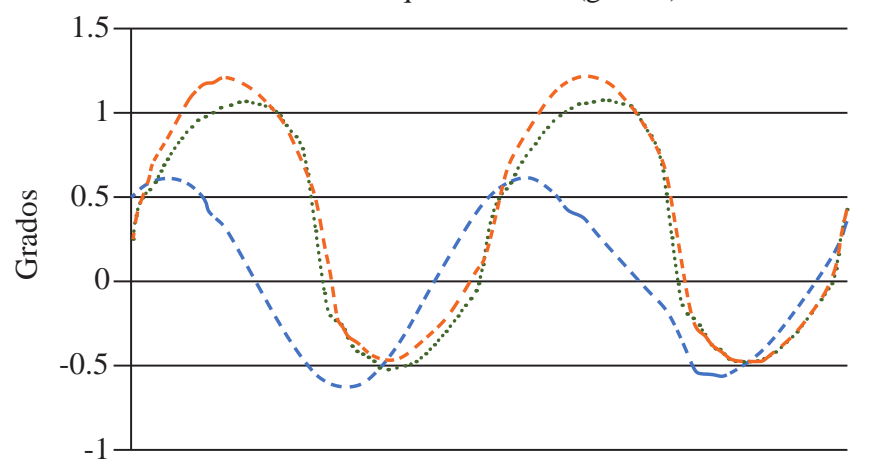

A

Torque (N-m)

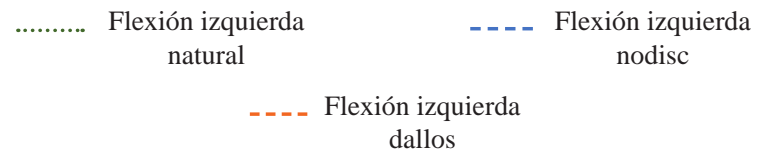

Flexión lateral derecha 400N (grados)

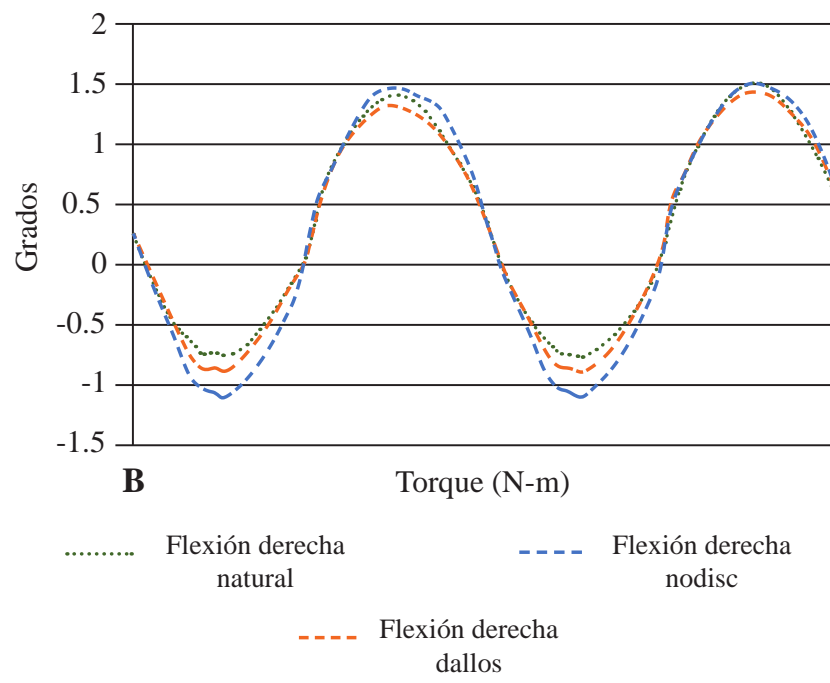

Figura 5: A) Muestra pérdida completa en la flexión lateral izquierda de la viscolestaticidad del disco posterior a su remoción del lado izquierdo, que es donde se realizó la discectomía más el aumento del rango de flexión para alcanzar el mismo torque que mostraron las condiciones con disco intacto y resección de disco más ligamentoplastía. B) Muestra cierta preservación de la distribución de fuerzas en la flexión derecha, probablemente debido a remanente del disco de este lado, pero también demostrando un mayor rango para la obtención del torque de $7.5 \mathrm{~N}-\mathrm{m}$.

diciones de segmento natural, postdiscectomía y postdiscectomía + ligamentoplastía fueron para la flexión $7.23^{\circ}, 8.65^{\circ}$ y $7.16^{\circ}$, respectivamente.

Para la extensión fueron $1.20^{\circ}, 1.14^{\circ}$ y $0.78^{\circ}$. En la flexión lateral derecha fueron de $10^{\circ}, 7^{\circ}$ y $7^{\circ}$, y la flexión lateral izquierda de $5.4^{\circ}, 6.6^{\circ}$ y $6^{\circ}$. Para la rotación derecha fueron de $1^{\circ}, 1.13^{\circ}$ y $1.21^{\circ}$ y para la izquierda $2^{\circ}, 2.2^{\circ}$ y $2.2^{\circ}$.

En promedio, en todos los movimientos con y sin carga de $400 \mathrm{~N}$, la disminución del espacio intervertebral después de la discectomía fue de $1 \mathrm{~mm}$, pero recuperó $0.5 \mathrm{~mm}$ después de la ligamentoplastía.

No hubo desplazamientos cizallantes significativos durante las tres condiciones.

\section{Discusión}

En la actualidad, la estabilización rígida o dinámica son opciones ampliamente utilizadas para el tratamiento de patologías espinales, en especial en el segmento lumbar y aquéllas relacionadas con patología discal. Se ha demostrado que la estabilidad vertebral tiene que ver con la altura del disco y la tensión ligamentaria anterior y posterior, por lo que se compromete necesariamente cuando hay enfermedad discal. Después de la discectomía, una instrumentación rígida como la que proporcionan los tornillos y barras provee de estabilidad total del segmento. Sin embargo, la rigidez del segmento estabilizado también se relaciona con la enfermedad de segmento adyacente en un tercio de los pacientes

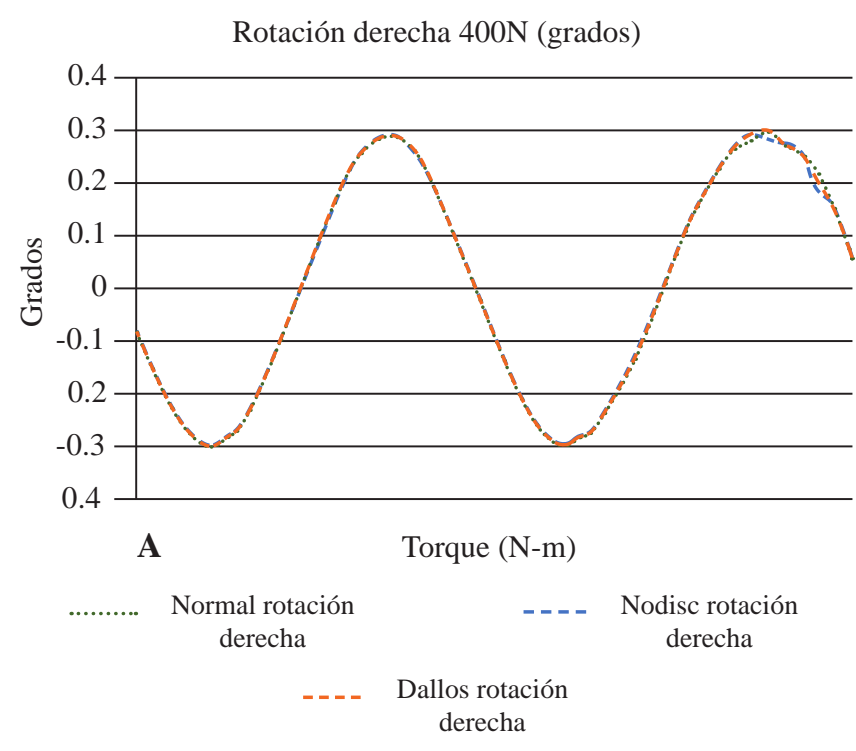

Rotación izquierda 400N (grados)

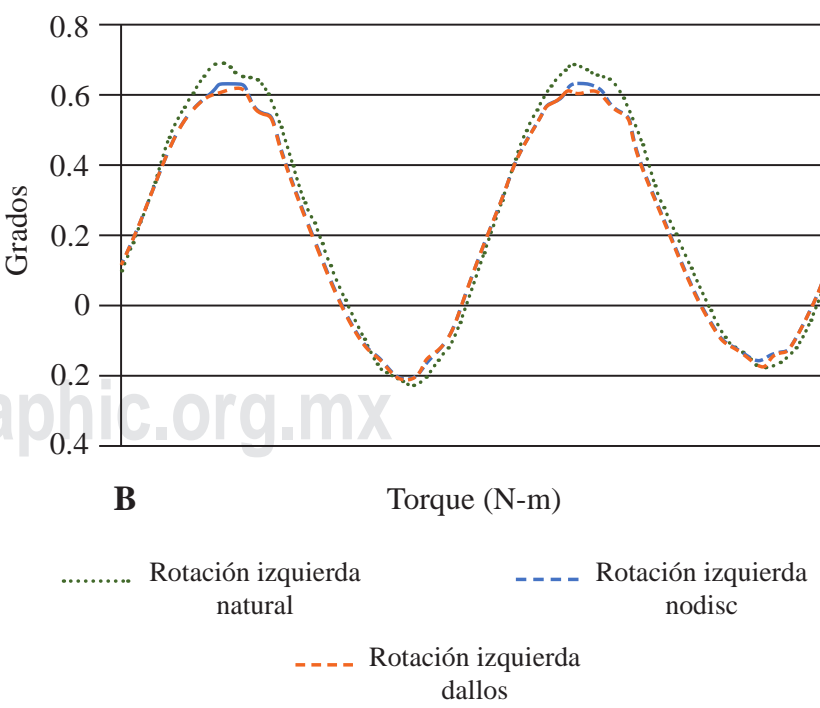

Figura 6: A) Se observa nuevamente restricción a la rotación por la preservación de una parte del disco intervertebral. B) Muestra aumento en la rotación izquierda a expensas de la discectomía de ese lado. 
en los que se practica. Por el contrario, la estabilización dinámica, aquélla que permite una rigidez relativa amén de permitir arcos de movilidad restringidos en los diferentes ejes de movimiento de la columna, ha mostrado una menor incidencia de enfermedad adyacente comparada con la estabilización rígida. En nuestro estudio encontramos que en la flexión con y sin carga y la extensión con carga del espécimen posterior a discectomía se demandó mayor rango de movilidad para alcanzar el torque deseado. Para la extensión con carga, la diferencia fue prácticamente nula entre las tres condiciones debido a la restricción mecánica de las apófisis espinosas al hacer contacto entre ellas bajo condiciones de carga y restringir el movimiento sin importar la ausencia del disco o la presencia de la ligamentoplastía. Sin embargo, el aplanamiento de las curvas para la condición sin disco y sin disco más ligamentoplastía sugiere que la carga sobre las apófisis espinosas y las articulaciones cigapofisarias será mayor y con un comportamiento brusco y no viscoelástico como lo provee el disco intacto. La flexión lateral derecha en todas las condiciones permitió más grados de libertad que la flexión a la izquierda, ya que el torque en este sentido inició antes que el contralateral, sugiriendo que las plataformas superior e inferior se pusieron en contacto con mayor facilidad por la ausencia del disco y el anillo fibroso, pues la discectomía fue realizada del lado izquierdo. La rotación axial derecha se mantuvo prácticamente sin diferencia en las tres condiciones con y sin carga; sin embargo, en rotación izquierda aumentó en aproximadamente un grado en todas las condiciones con y sin carga, lo que sugiere nuevamente que la discectomía realizada en la parte posterior e izquierda otorgó mayor inestabilidad hacia ese mismo lado, quizá por la ausencia de parte del anillo fibroso ipsilateral.

Basado en estos datos, la inestabilidad que produce la resección del disco es evidente, sobre todo del lado donde se realizó la discectomía. La ligamentoplastía posterior a la discectomía demostró sobre todo en flexión y extensión que puede conferir mayor rigidez al segmento espinal similar al del segmento natural intacto y al mismo tiempo conservar sus grados de movilidad, por lo que biomecánicamente sugiere que la fijación vertebral es en efecto dinámica y estable al menos en el eje de flexoextensión. Los cambios mecánicos en la flexión lateral derecha y rotación axial izquierda se explican por la ausencia del anillo fibroso en la parte posterolateral izquierda y no mostraron diferencias significativas cuando se compara en las tres condiciones. Referente a la translación axial del espacio intervertebral, la ligamentoplastía demostró que la altura inicial en el segmento natural intacto que perdió $0.5 \mathrm{~mm}$ posterior a discectomía, logró recuperarse posterior a la ligamentoplastía con el ligamento de poliéster.

Un hallazgo interesante fue el comprobar que la biomecánica segmentaria lumbar afecta principalmente a la flexión lateral y rotación del lado donde se realiza la discectomía, sugiriendo que, a pesar de la estabilización con la ligamentoplastía, la transmisión de la fuerza se verá afectada en ese lado. Teóricamente la resección del dis- co mediante una fenestración se puede realizar de manera adecuada; sin embargo, la distribución de las fuerzas del lado donde se extrae el disco indica que hay un compromiso mayor cuando se compara con el comportamiento biomecánico del lado contrario a la discectomía, esto quizá porque algún remanente discal permanece en el espacio intervertebral al no ser retirado completamente y dejando una parte del mismo que aun proporcionará la transmisión de las fuerzas con un comportamiento viscoelástico. También, el aumento de las fuerzas para el lado izquierdo ante la flexión lateral izquierda y la rotación axial ipsilateral indican que la distribución de la fuerza se vuelve más pronunciada sin su componente viscoelástico, transmitiendo estrés a las estructuras posteriores que son las que cargarán estas fuerzas como lo son las articulaciones facetarias, el complejo ligamentario interespinoso y las apófisis espinosas.

Estudios que cuenten con más especímenes, incluyendo un espécimen humano, son deseables para corroborar estos resultados. Un estudio que incluya fatiga cíclica de la estabilización dinámica con la fibra es deseable para poder garantizar que la estabilidad obtenida es sostenible a lo largo de los años de vida del paciente.

\section{Conclusiones}

La ligamentoplastía con fibra de poliéster puede preservar los movimientos multiaxiales de la columna posterior a la realización de la discectomía, especialmente en la flexión y la extensión.

La recuperación de la altura del espacio intervertebral observada durante el estudio sugiere que la ligamentoplastía confiere estabilidad al reajustar la tensión ligamentaria anterior y posterior del segmento vertebral lumbar.

Referencias

1. Panjabi MM. Clinical spinal instability and low back pain. $J$ Electromyogr Kinesiol. 2003; 13(4): 371-9.

2. Panjabi MM. The stabilizing system of the spine: Part I. function, dysfunction, adaptation, and enhancement. J Spinal Disord. 1992; 5(4): 383-9.

3. Sengupta DK. Dynamic stabilization devices in the treatment of low back pain. Orthop Clin North Am. 2004; 35(1): 43-56.

4. Posner I, White AA, Edwards WT, Hayes WC. A biomechanical analysis of the clinical stability of the lumbar and lumbosacral spine. Spine (Phila Pa 1976). 1982; 7: 374-89.

5. Kirkaldy-Willis WH, Farfan HF. Instability of the lumbar spine. Clin Orthop Relat Res. 1982; 165: 110-23.

6. Chan AK, Sharma V, Robinson LC, Mummaneni P V. Summary of guidelines for the treatment of lumbar spondylolisthesis. Neurosurg Clin N Am [Internet]. 2019; 30(3): 353-64. Available from: https://doi. org/10.1016/j.nec.2019.02.009

7. Kanayama M, Togawa D, Hashimoto T, Shigenobu K, Oha F. Motionpreserving surgery can prevent early breakdown of adjacent segments. J Spinal Disord Tech. 2009; 22(7): 463-7.

8. Alpízar-Aguirre A, Guevara-Alvarez A, Rosales-Olivares LM, ZárateKalfópulos B, Sánchez-Bringas G, Reyes-Sánchez A. Estabilización dinámica interespinosa versus fijación transpedicular y artrodesis en el tratamiento del conducto lumbar estrecho en pacientes de 45 a 65 años de edad. Acta Ortop Mex. 2012; 26(6): 347-53. 
9. Okuda S, Nagamoto Y, Matsumoto T, Sugiura T, Takahashi Y, Iwasaki M. Adjacent segment disease after single segment posterior lumbar interbody fusion for degenerative spondylolisthesis. Spine. 2018; 43(23): E1384-8.

10. Panjabi MM. The stabilizing system of the spine. Part II. Neutral zone and instability hypothesis. J Spinal Disord. 1992; 5(4): 390-6; discussion 397.

11. Yue JJ, Timm JP, Panjabi MM, Jaramillo-de la Torre J. Clinical application of the Panjabi neutral zone hypothesis: the Stabilimax NZ posterior lumbar dynamic stabilization system. Neurosurg Focus. 2007; 22(1): E12.

12. David G, Marsh J, Mahir S, Leyte A. A prospective randomised controlled trial to assess the efficacy of dynamic stabilisation of the lumbar spine with the Wallis ligament. Eur Spine J. 2014; 23(10): 2156-60.

13. Félix-Garza R, Lagarda-Cuevas J, Chávez-Maqueda MF, LadewigBernáldez GI. Espondilolistesis lumbar degenerativa de un solo segmento tratada con espaciador interespinoso. Acta Ortop Mex. 2014; 28(2): 88-94.
14. Wilke HJ, Drumm J, Haussler K, MacK C, Kettler A. Biomechanik der interspinosen Platzhalter. Der Orthopade. 2010; 39(6): 565-72.

15. Beckmann A, Nicolini LF, Grevenstein D, Backes H, Oikonomidis S, Sobottke R, et al. Biomechanical in vitro testof a novel dynamic spinal stabilization system incorporating polycarbonate urethane material under physiological conditions. J Biomech Eng. 2020; 142(1): 011005.

16. Senegas J, Etchevers JP, Vital JM, Baulny D, Grenier F. Recalibration of the lumbar canal, an alternative to laminectomy in the treatment of lumbar canal stenosis. Rev Chir Orthop Reparatrice Appar Mot. 1988; 74(1): 15-22.

17. Fehlings MG, Chua SY. Interspinous ligamentoplasty. J Neurosurg Spine. 2010; 13(1): 24-5.

18. Hong SW, Lee HY, Kim KH, Lee SH. Interspinous ligamentoplasty in the treatment of degenerative spondylolisthesis: midterm clinical results. clinical article. J Neurosurg Spine. 2010; 13(1): 27-35.

19. Rosales-Olivares LM, Alpízar-Aguirre A, Miramontes-Martínez V, Zárate-Kalfópulus B, Reyes-Sánchez A. Estabilización dinámica interespinosa en discectomía lumbar. Seguimiento de cuatro años. Cir Cir. 2010; 78(6): 495-9. 